\title{
Cytotoxicity Induced by Bismuth Subcitrate in Giardia lamblia Trophozoites
}

\author{
M. C. SOUSA* and J. POIARES-DA-SILVA \\ Laboratório de Microbiologia e Parasitologia and Centro de Estudos Farmacêuticos da Faculdade de \\ Farmácia, Universidade de Coimbra, 3030 Coimbra, Portugal
}

\begin{abstract}
The cytotoxicity of colloidal bismuth subcitrate (CBS) was investigated in cultured Giardia lamblia trophozoites on the basis of cell attachment, morphology and viability studies. The effects on cell membrane integrity were evaluated by the permeability to trypan blue, and the morphological alterations were studied by phase-contrast microscopy and transmission electron microscopy. Our data show that although CBS induced loss of cellular viability (morphological and regrowth studies), the cell membrane permeability was not altered. The attachment of $G$. lamblia trophozoites to culture vials was rapidly disrupted by CBS. Ultrastructural observations revealed that this drug promoted modifications on the cell shape, displacement of nucleus and of cytoskeletal structures, pronounced cytoplasmic vacuolization, dorsal and ventral protuberance of cytoplasmic membrane and heavy deposition of electron-dense precipitates in adhesive disc, nuclear membrane and cytoplasmic components. In contrast, membranes and microtubules were apparently undamaged. Some of these results suggest that the cytotoxicity of CBS to G. lamblia cultures is the result of its binding to cytoskeletal components. As far as we know, this work is the first demonstration that bismuth subcitrate could kill a human protozoan parasite suggesting its potential in the therapy of giardiasis. (C) 1999 Elsevier Science Ltd. All rights reserved
\end{abstract}

Keywords: Giardia lamblia; bismuth subcitrate; ultrastructure; viability; adherence.

Abbreviations: CBS $=$ bismuth subcitrate; HBSS $=$ Hanks' balanced salt solution.

\section{INTRODUCTION}

Giardia lamblia is a binucleated flagellated protozoan that causes infection of the small intestine that may give rise to diarrhoea with or without malabsorption. This parasite is endemic throughout the world and it is one of the most frequently found intestinal parasites in children living in developing countries. Although various drugs have been available for several decades to treat this infection, none is entirely satisfactory due to the high incidence of undesirable side-effects, frequent relapses and potential carcinogenic effects. Therefore, there is an obvious need for alternative antigiardial agents.

Recent studies have shown the occurrence of gastric giardiasis concomitant with intestinal giardiasis and a significant association of $G$. lamblia with Helicobacter pylori in gastric biopsies (Sanad et al., 1996). It is well known that H. pylori is a Gramnegative bacterium causing gastric mucosal infection and that is susceptible to a wide range of antimicrobial agents, namely bismuth compounds.

*Corresponding author at: Couraça dos Apóstolos, $\mathrm{n}^{\mathrm{o}}$ 51, R/C, Faculdade de Farmácia da Universidade de Coimbra, 3030 Coimbra, Portugal.
These findings prompted us to study the interaction of colloidal bismuth subcitrate (CBS), used for the treatment of gastric and duodenal ulcers (Lambert et al., 1992), with G. lamblia trophozoites. The bismuth antimicrobial action is complex and still poorly understood (Domenico et al., 1996, Lambert and Midolo, 1997). Thus, in this work we studied the in vitro effects of CBS on the viability, adherence and morphology of the parasite to evaluate its potential value in the chemotherapy of giardiasis and to investigate the drug action mechanism(s).

\section{MATERIALS AND METHODS}

\section{Chemicals}

Bile bovine, L-cysteine, L-ascorbic acid and ferric ammonium citrate were obtained from Sigma Chemical Co.; casitone and yeast extract were obtained from Difco Laboratories and bovine serum and antibiotic solution were obtained from Biochrom K.G. We are indebted to Yamanouchi Pharma for the gift of bismuth subcitrate. 


\section{Parasites and cultures}

Giardia lamblia (WB strain, originally from a patient with chronic diarrhoea) was obtained from The American Type Collection (Rockville, MD, USA) (ATCC 30957). Trophozoites were routinely culture in $10 \mathrm{ml}$ of Diamond's TYI-S-33 medium modified by Keister (1983) and supplemented with a commercial penicillin-streptomycin-amphotericin solution. After 2 days, the cultures were harvested by cooling the culture vials, at $4^{\circ} \mathrm{C}$, and centrifugation at $1500 \mathrm{rpm}$ for $10 \mathrm{~min}$. Trophozoites were washed three times in Hanks' balanced salt solution (HBSS, pH 7.1) and cell count was performed in a haemocytometer (Neubaeur cell-counter chamber). These cells were used as inoculum for studying the effects of CBS on viability, morphology and adherence of $G$. lamblia trophozoites.

\section{CBS toxicity studies}

An inoculum of $10^{6}$ trophozoites was exposed to CBS $(1-10 \mathrm{mg} / \mathrm{ml})$ in HBSS $(\mathrm{pH} 7.1)$ for $2 \mathrm{hr}$, at $37^{\circ} \mathrm{C}$, using $10-\mathrm{ml}$ polystyrene screw-capped vials. Control experiments were performed in similar experimental conditions without the drug, in the presence only of the drug solvent (HBSS, pH 7.1). After incubation, the vials were cooled at $4{ }^{\circ} \mathrm{C}$ and the suspension centrifuged at $1500 \mathrm{rpm}$, for $10 \mathrm{~min}$. Trophozoites were washed three times in cold HBSS and the cell pellets were resuspended in HBSS. Trophozoites viability were directly determined by phase-contrast microscopy, counting the live and dead cells in a haemocytometer (Hill et al., 1986). Parasites were considered viable if they had a characteristic pear-shaped structure, flagellar motility, normal architecture of ventral disc and refractory quality. To assess cell membrane integrity, trophozoite suspensions were incubated on ice with an equal volume of $0.4 \%$ trypan blue, and loaded into a haemocytometer for viable cell counting (Aley et al., 1994). The regrowth assay, based on the ability of viable $G$. lamblia trophozoites to multiply and growth in fresh culture medium after being exposed to a lethal agent, has been extensively used for the determination of parasite viability (Cedillo-Reviera and Muñoz, 1992; Farbey et al., 1995; Hempill et al., 1996; Hill et al., 1996). The reduction of the number of attached trophozoites, after regrowth, as an index of reduction of viability. Thus, $30 \mu \mathrm{l}$ of each incubation were sub- cultivated for $48 \mathrm{hr}$, at $37^{\circ} \mathrm{C}$, in $10 \mathrm{ml}$ of fresh TYIS-33 medium supplemented with antibiotics. Subsequently, the medium was removed by vacuum aspiration and the adherent trophozoites were detached by incubation for $10 \mathrm{~min}$, at $4{ }^{\circ} \mathrm{C}$, in icecold HBSS. Finally, the number of adherent trophozoites was determined microscopically using a haemocytometer. The experiments were performed three times and the results were expressed as a percentage of viability and of number of attached cells after $48 \mathrm{hr}$.

\section{Adherence study}

The direct effect of CBS on adherence of G. lamblia trophozoites was evaluated as previously described by Edlind and Hang (1990). An inoculum of $5 \times 10^{5}$ cells were distributed into vials $(10 \mathrm{ml})$ and were incubated for $2 \mathrm{hr}$, at $37^{\circ} \mathrm{C}$, in TYI-S-33 medium without antibiotics. Preliminary studies showed that the maximum number of cells was attached at this time. The medium with unattached cells was discarded and replaced with prewarmed medium, with and without CBS $(1-10 \mathrm{mg} / \mathrm{ml})$, and the cultures were incubated for $2 \mathrm{hr}$, at $37^{\circ} \mathrm{C}$. Adherent cells were dislodged by a 10 -min incubation in ice-cold HBSS, and the number of attached cells were determined microscopically using a haemocytometer. The experiments were performed three times and the results were expressed as number of attached cells.

\section{Transmission electron microscopy}

Samples were treated as reported previously (Chávez et al., 1986). Cell pellets were fixed with $2.5 \%$ glutaraldehyde in $0.1 \mathrm{M}$ sodium cacodylate buffer ( $\mathrm{pH} 7.0$ ), post-fixed in $1 \%$ osmium tetroxide and uranyl acetate, dehydrated in ethanol and in propylene oxide and embedded in Epon 812 (TAAB 812) resin. Ultra-thin sections were stained with lead citrate and uranyl acetate and observed in a JOEL $100 \mathrm{~S}$ electron microscope.

\section{RESULTS}

\section{Viability studies}

CBS induces loss of cellular viability but does not change the membrane integrity, as evaluated by the permeability of trophozoites to trypan blue (Fig. 1a). The dose-response curve indicates that

Plate 1. (a-f) Transmission electron micrographs of Giardia lamblia trophozoites (a) Untreated parasites (20000×); (b-f) Trophozoites exposed to CBS for $2 \mathrm{hr}$, at $37^{\circ} \mathrm{C}$. (b) Note the grotesque modification of cell shape with dorsal protuberance (arrows), displacement of nucleus and fragmentation and displacement of adhesive disc (16000×). (c) Various dorsal protuberances (arrows) and displacement of axonemal microtubules (11500×). (d) Some cells show absence of adhesive disc, displacement of axonemal microtubule with the cytoplasm less electron-dense $(23000 \times)$. (e) Note electron-dense precipitates on adhesive disc and on nuclear membrane (arrows) (68700×). (f) Pronounced vesiculation (V), blebbing (B) and electron-dense precipitates are also found on adhesive disc (arrows) $(11500 \times)$. ( $\mathrm{N}=$ nucleus; $\mathrm{AD}=$ adhesive disc; $\mathrm{FA}=$ axonemal microtubules). 

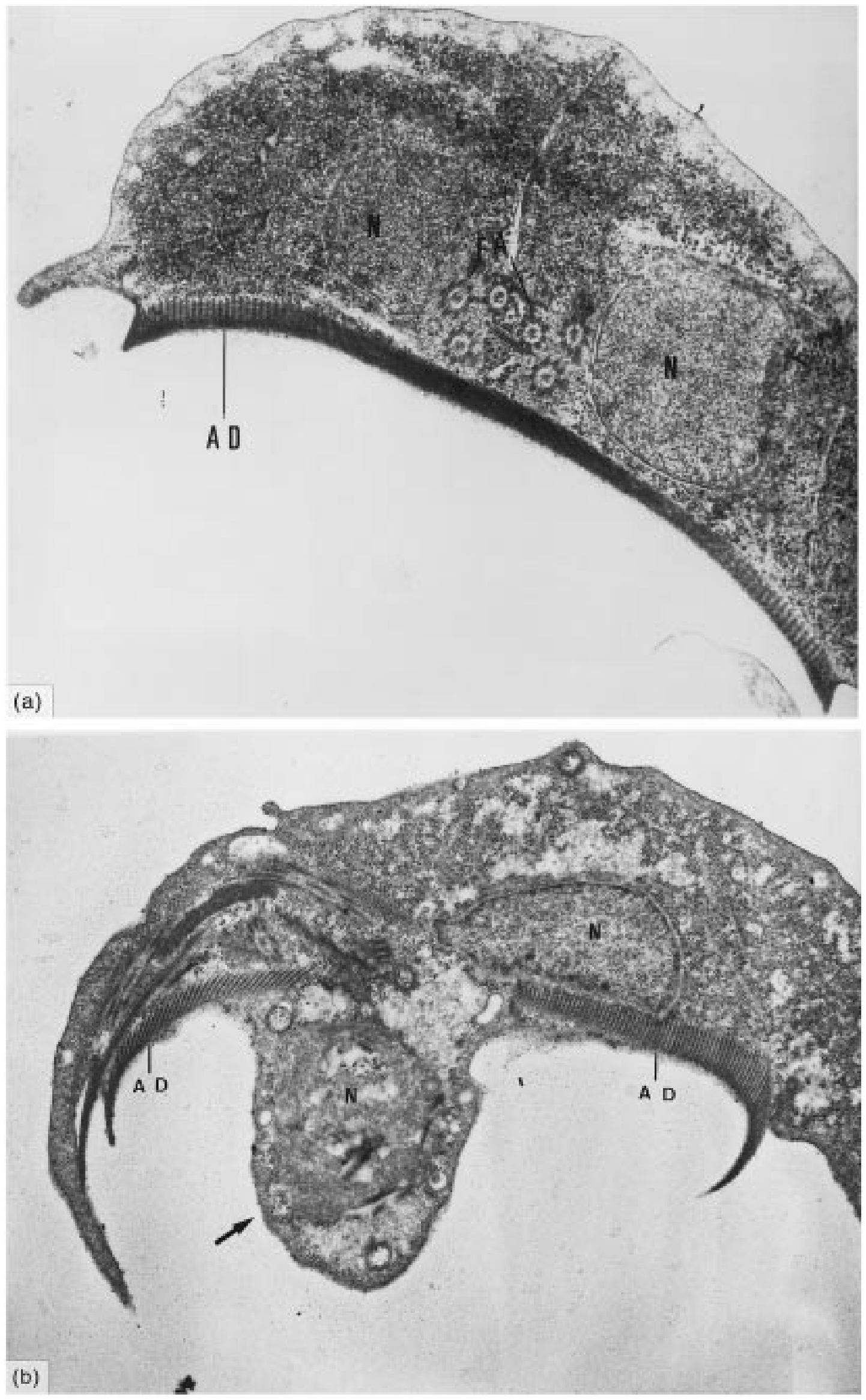

Plate 1 [continued overleaf] 

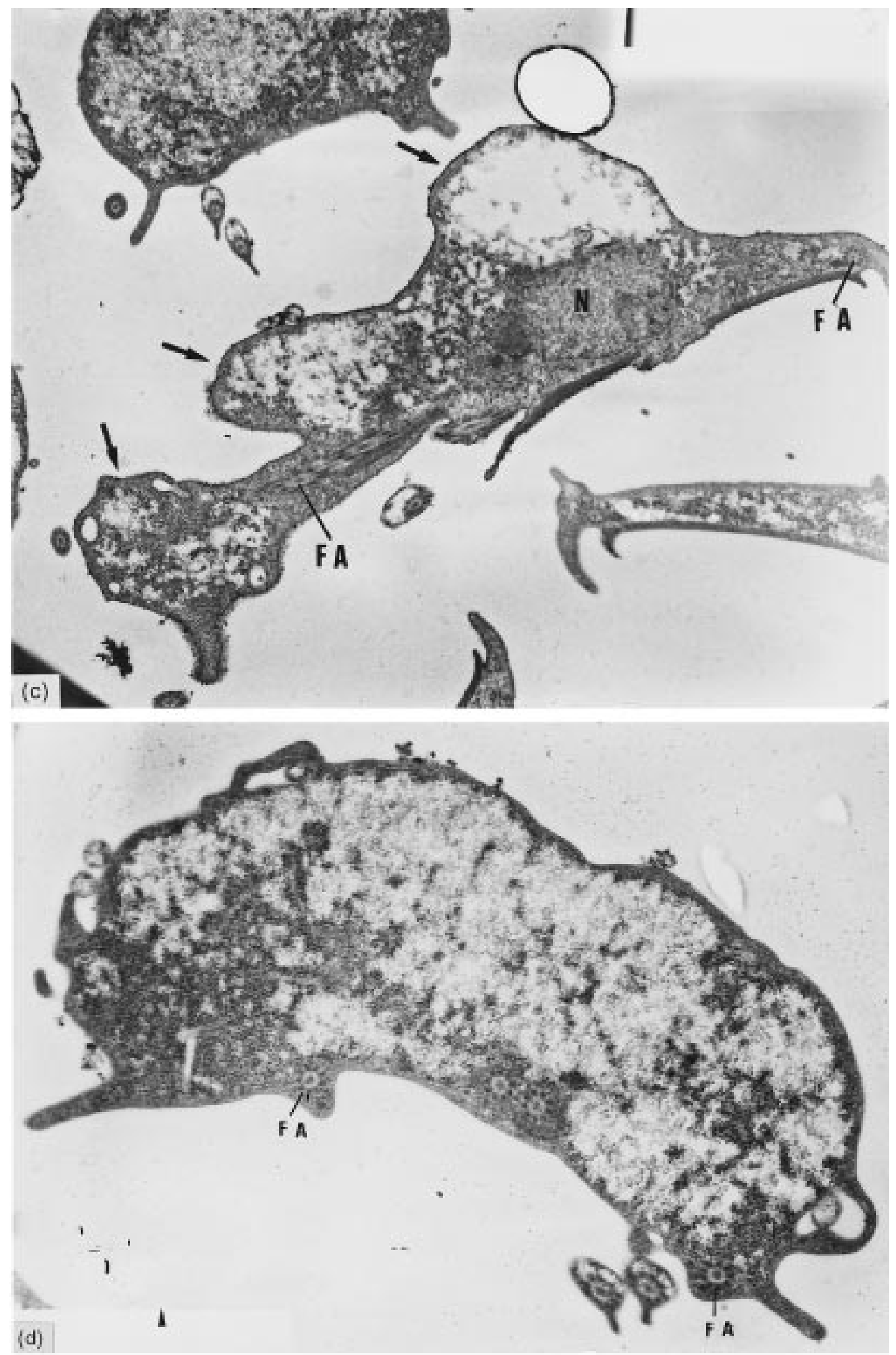

Plate 1 [continued] 


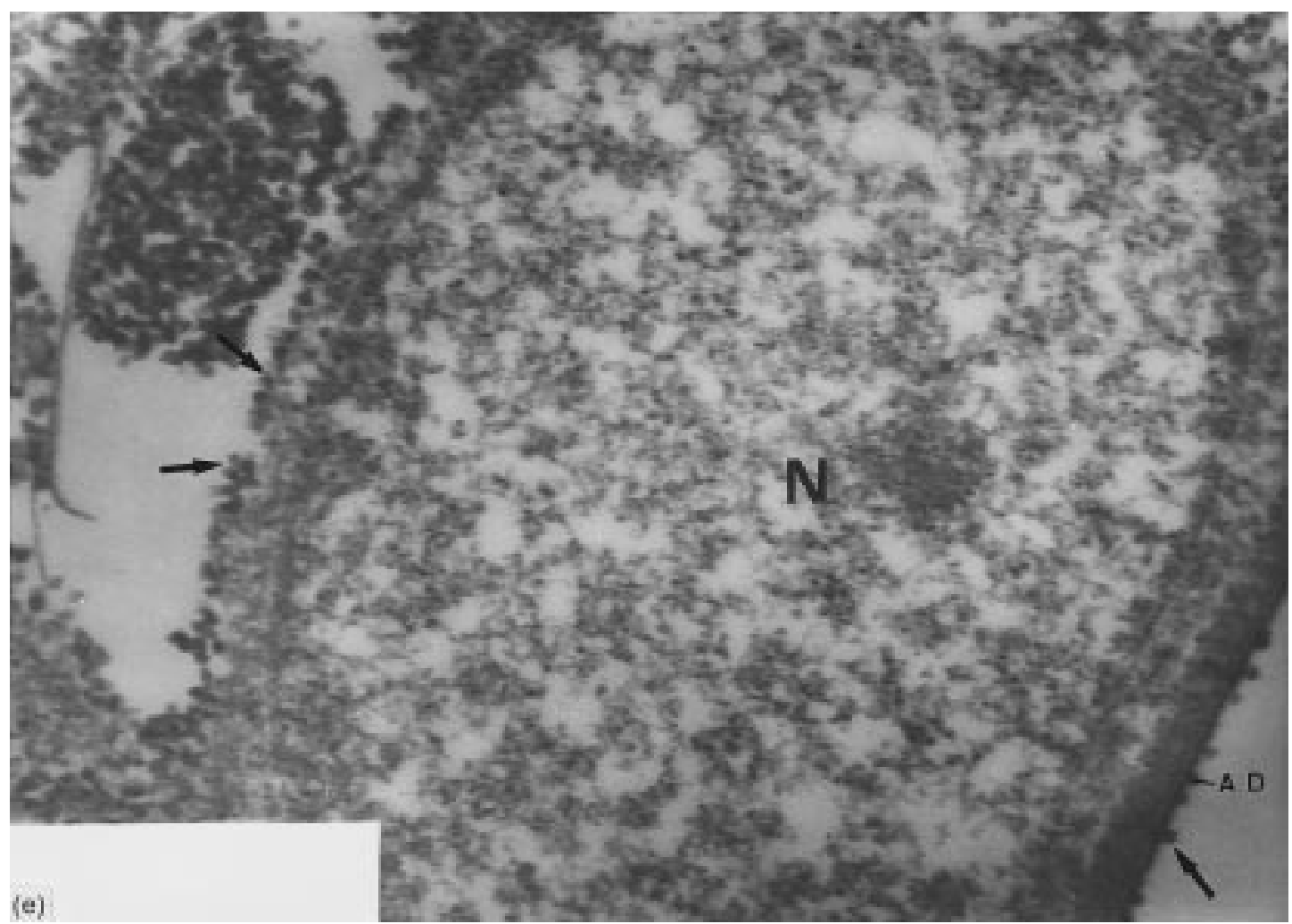

Plate 1 [continued overleaf]

CBS $(1-10 \mathrm{mg} / \mathrm{ml})$ induced the death of $11-90 \%$ of $G$. lamblia trophozoites as evaluated by the morphological criteria (Fig. 1a). The killed parasites show, by phase contrast microscopy, a typical pearshaped structure, refractory quality and loss of flagellar motility (not shown).

The results, obtained by regrowth assays, point to a $22-100 \%$ decreases in the number of adherent cells over $48 \mathrm{hr}$, after exposure to CBS (1-10 mg/ $\mathrm{ml}$ ) (Fig. 1a). These results confirm the lethal effect of CBS and are correlate very well with those obtained by morphological criteria.

\section{Adherence inhibition}

G. lamblia trophozoites can attach to in vitro cultured cells as well as to glass and to a variety of artificial substrates (Kulda and Nohynková, 1995). Considering that inhibition of its attachment may have therapeutic potential, it was of interest to study the ability of CBS to promote the G. lamblia detachment. Figure 1(b) shows that $G$. lamblia attachment to plastic culture vials is strongly inhibited by CBS.

\section{Ultrastructural effects of CBS on trophozoites}

Transmission electron microscopy of untreated $G$. lamblia trophozoites shows that these cells have a typical cytoskeleton with four pairs of flagella and adhesive disc, nucleus, and cytoplasmic membrane (Plate 1a). CBS induces morphological changes in cells after exposure for $2 \mathrm{hr}$, at $37^{\circ} \mathrm{C}$. Trophozoites show modifications of cell shape, dorsal and ventral protuberance, displacement of nucleus and of cytoskeletal structures and fragmentation of adhesive disc (Plate 1b,c). Frequently, flagella appear intact but irregularly dispersed in the cytoplasm (Plate 1d). Moreover, electron dense deposits are much more evident on adhesive disc, nuclear membrane and other cytoplasmic components in trophozoites treated with CBS (Plate 1e,f). In some cells we can see pronounced cytoplasmic vesiculation and vacuolization as well as surface protrusions (blebs) (Plate 1f).

\section{DISCUSSION}

Bismuth salts have been used in the treatment of gastric and duodenal ulcers, and were the first antibacterial drugs used in the eradication of $H$. pylori infection (Coghlan et al., 1987). Considering the occurrence of $G$. lamblia in the gastric mucosa and the association of $G$. lamblia with $H$. pylori in gastric biopsies, we studied the cytotoxicity of bismuth 


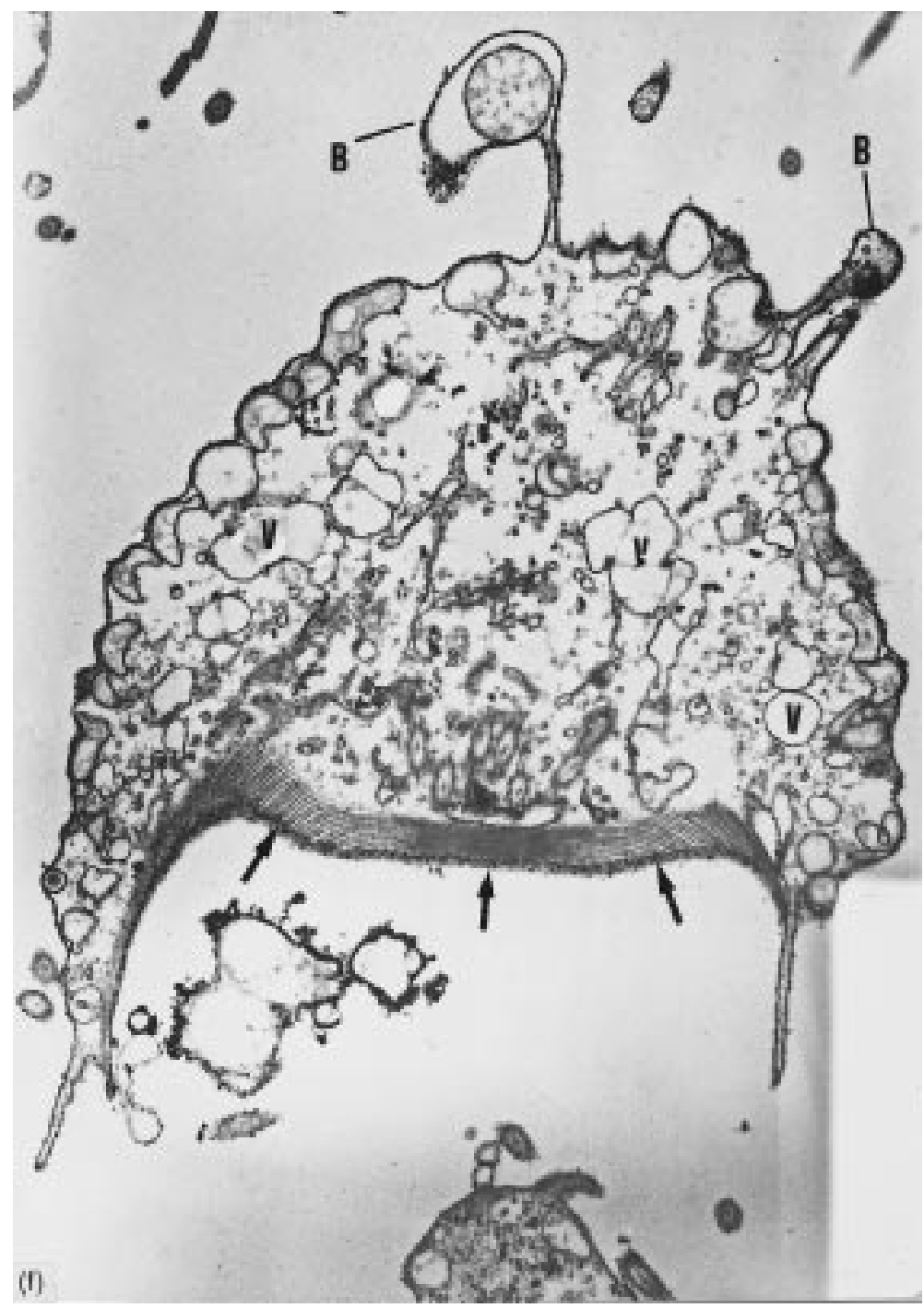

Plate 1 [continued $]$

subcitrate in G. lamblia trophozoites to evaluate its potential use in therapy of giardiasis.

Adherence of $G$. lamblia trophozoites to enterocytes is essential for colonization and is considered to play a crucial role in the pathogenesis of giardiasis (Farthing et al., 1997). Therefore, inhibition of parasite attachment may have therapeutic potential. The present study demonstrates that CBS inhibits the G. lamblia trophozoites attachment in vitro and induces loss of cell viability. Although the permeability of cells treated with CBS does not change, the lethal effect of CBS was demonstrated by the regrowth assay. Our data have additional implications regarding the clarification of drug action mechanism(s). Considering that microtubules are the major components of the adhesive disc, which mediates the cell attachment, and of the cytoskele- ton, which is essential for maintaining the cell structure (Kulda and Nohyhová, 1995), our results suggest an interaction of bismuth subcitrate with $G$. lamblia microtubules that could be the basis of its antigiardial action. This is supported by ultrastructural observations and adherence studies. Actually, trophozoites treated with CBS show modifications of cell shape, fragmentation and displacement of adhesive disc components, electron-dense deposits in adhesive disc, irregular dispersion of axonemal microtubules in the cytoplasm and detachment of G. lamblia cells.

It has been reported that the important feature of antigiardial chemotherapy may be the concentration at the site of infection rather than the drug plasma levels (Reynoldson et al., 1992), and that bismuth subcitrate is a safe drug which exerts local effects 
(a)
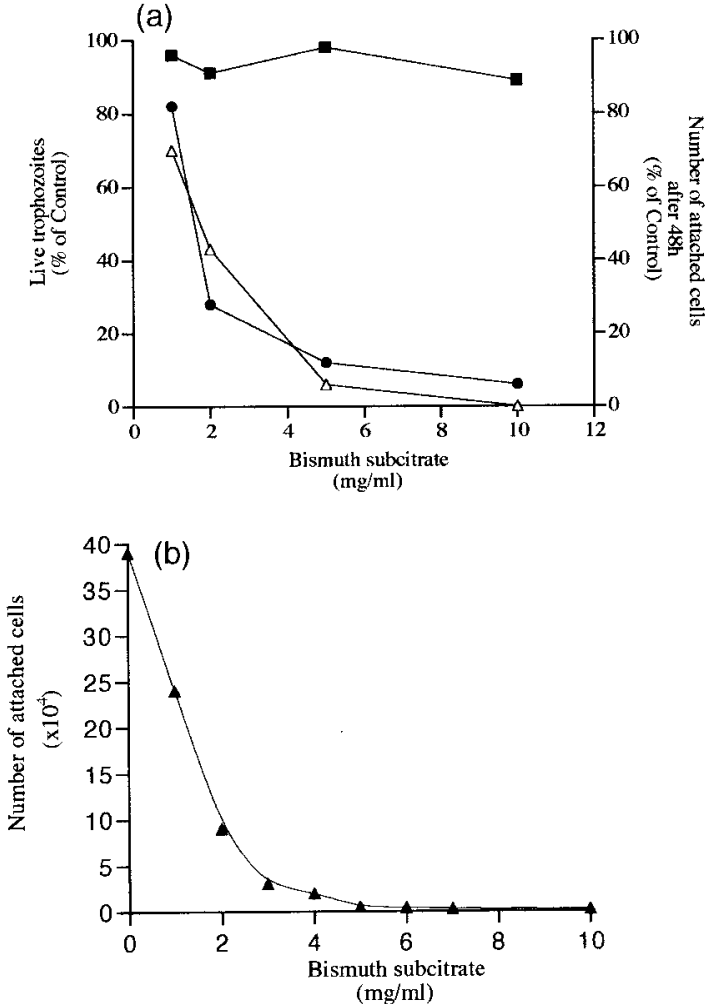

Fig. 1. (a) Killing of Giardia lamblia trophozoites by bismuth subcitrate. Trophozoites were incubated with various concentrations of CBS for $2 \mathrm{hr}$ at $37^{\circ} \mathrm{C}$, and trophozoite viability was determined by trypan blue exclusion $(\square)$, morphology ( $)$ and regrowth assay (number of attached cells after $48 \mathrm{hr})(\triangle$ ). (b) Effect of bismuth subcitrate in adherence of G. lamblia trophozoites to the culture vessel after $2 \mathrm{hr}$ of drug exposure. These dose-response curves are typical of three independent experiments and error bars are not indicated, since for the most part, they are encompassed by the size of the symbols.

on gastroduodenal mucosa. In therapy regimens for the treatment of gastric and duodenal ulcer the usual daily dose of CBS is $480 \mathrm{mg}$ and the treatment period is usually 2 wk (Reynolds, 1996; Robinson et al., 1997). Considering these findings, our data suggest that CBS can be effective in the treatment of giardiasis and may be particularly useful in associated $G$. lamblia and $H$. pylori infections.

Acknowledgements - We thank Professor Vasco Bairos (Institute of Histology, Faculty of Medicine, Coimbra) for the loan of the electron microscope. This work was partially supported by PRODEP II.

\section{REFERENCES}

Aley B. S., Zimmerman M., Hetsko M., Selsted M. E. and Gillin F. D. (1994) Killing of Giardia lamblia by cryptdins and cationic neutrophil peptides. Infection and Immunity 62, 5397-5403.

Cedilo-Reviera R. and Muñoz O. (1992) In-vitro susceptibility of Giardia lamblia to albendazole, mebendazole and other chemotherapeutic agents. Journal of Medical Microbiology 37, 221-224.

Chávez B., Knaippe F., González-Mariscal L. and Martinez-Pulomo A. (1986) Giardia lamblia: electrophysiology and ultrastructure of cytopathology in culture epithelial cells. Experimental Parasitology 61, 379 389.
Coghlan J. G., Gilligan D. and Humphires H. (1987) Campylobacter pylori and recurrence of duodenal ulcers - a 12 month follow up study. Lancet ii, 1109-1111.

Domenico P., Reich J., Madonia W. and Cunha B. A. (1996) Resistance to bismuth among Gram-negative bacteria is dependent upon iron and its uptake. Journal of Antimicrobial Chemotherapy 38, 1031-40.

Edlind T. D. and Hang T. L. (1990) Activity of the anthelmintic benzimidazoles against Giardia lamblia in vitro. Journal of Infections Diseases 162, 1408-1411.

Farbey M. D., Reynoldson J. A. and Thompson R. C. A. (1995) In vitro drug susceptibility of 29 isolates of Giardia duodenalis from humans as assessed by an adhesion assay. International Journal of Parasitology 25, 593-599.

Farthing M. J. G. (1997) The molecular pathogenesis of giardiasis. Journal of Pediatric Gastroenterology and Nutrition 24, 79-88.

Hempill A., Stager S., Gottstein B. and Muller N. (1996) Electron microscopical investigation of surface alterations on Giardia lamblia trophozoites after exposure to a cytotoxic monoclonal antibody. Parasitology Research 82, 206-210.

Hill D. R., Pohl R. and Pearson R. D. (1986) Giardia lamblia: A culture method for determining parasite viability. American Journal of Tropical Medicine and Hygiene 35, 1129-1133.

Keister B. D. (1983) Axenic culture of Giardia lamblia in TYI-S-33 medium supplemented with bile. Transactions of the Royal Society of Tropical Medicine and Hygiene 77, 487-488. 
Kulda J. and Nohyhová E. (1995) Giardia in humans and animals. In Parasitic Protozoa. Edited by Julius P. Kreier, pp. 229-268. Academic Press.

Lambert J. R., Lin S. K., Shembri M., Nicholson L. and Mclean A. J. (1992) Comparison of different preparations of Denol in healing of duodenal ulcer and Helicobacter pylori eradication. Hellenic Journal of Gastroenterology 5, 190.

Lambert J. R. and Midolo P. (1997) The actions of bismuth in the treatment of Helicobacter pylori infection. Alimentary Pharmacology and Therapy 11, 27-33.

Reynolds J. E. F. (1996) Martindale: The Extra Pharmacopoeia, 31st edn, pp. 1206-1207. The Pharmaceutical Press.
Reynoldson J. A., Thompson R. C. A. and Meloni B. P. (1992) The potential and possible mode of action of the benzimidazoles against Giardia and other protozoa. Journal of Pharmacological Medicine 2, 35-50.

Robinson D. M., Abdel Rahman S.M. and Nahata M. C. (1997) Guidelines for the treatment of Helicobacter pylori in pediatric population. Annals of Pharmacotherapy 31, 1247-1249.

Sanad M. M., Darwish R. A., Nasr M. E., Gammal N.E:. and Emare M. W. (1996) Giardia lamblia and chronic gastritis. Journal of the Egyptian Society of Parasitology 26, 481-495. 